\title{
Combination Venetoclax and Rituximab: A Novel Approach to Gastrointestinal Hemorrhage from Acquired Von Willebrand Syndrome in the Setting of Relapsed Chronic Lymphocytic Leukemia
}

\author{
Heald J T ${ }^{1 *}$ and Oleszewski RT ${ }^{2}$ \\ ${ }^{1}$ Department of Internal Medicine, Eisenhower Army \\ Medical Center, USA \\ ${ }^{2}$ Department of Hematology and Oncology, Eisenhower \\ Army Medical Center, USA \\ *Corresponding author: Heald JT, Department of \\ Internal Medicine, Eisenhower Army Medical Center, 300 \\ Hospital Rd, Fort Gordon, Ga 30905, USA
}

Received: September 20, 2021; Accepted: October 14, 2021; Published: October 21, 2021

\begin{abstract}
Congenital Von Willebrand Disease (VWD) is the most common bleeding diathesis in humans with a prevalence of one percent in the general population. However, acquired Von Willebrand Syndrome (aVWS) is a rare coagulopathy that has been reported to be associated with numerous conditions and occurs through a variety of mechanisms. In addition to causing bleeding diathesis due to coagulopathy, there is evidence that demonstrates Von Willebrand's Factor (VWF) deficiency leads to aberrant blood vessel formation. Treatment for this disease is directed at stabilization of associated hemorrhage and correction of underlying etiology. We present the case of a patient with aVWS disease due to relapse of Chronic Lymphocytic Leukemia (CLL) complicated by gastrointestinal hemorrhage from gastric and small bowel angiodysplasia treated with rituximab and venetoclax, previously unreported in the medical literature.
\end{abstract}

Keywords: Acquired Von Willebrand Syndrome; Chronic Lymphocytic Leukemia; Angiodysplasia; Rituximab; Venetoclax

\section{Abbreviations}

VWD: Von Willebrand Disease; aVWS: acquired Von Willebrand Syndrome; VWF: Von Willberand Factor; CLL: Chronic Lymphocytic Leukemia; ISTH: International Society on Thrombosis and Haemostasis; DDAVP: Desmopressin; IVIG: Intravenous Immune Globulin

\section{Introduction}

Congenital Von Willebrand disease is the most common bleeding diathesis in humans with a prevalence of approximately 1 percent with 1 in 10,000 to 1 in 100,000 displaying symptoms [1,2]. However, aVWS is a rare coagulopathy with an unknown true prevalence. Approximately 700 reported cases exist in the medical literature since first being described in 1968 [3]. Originally described in a patient with Systemic Lupus Erythematosus, aVWS has subsequently been reported in the setting of various malignancies, autoimmune disorders, severe aortic stenosis and left ventricular assist devices (LVAD) [4]. There is also evidence that deficiencies in Von Willebrand factor can lead to neovascularization notably angiodysplasia [5-8]. This phenomena has been most widely described in patients with LVADs or severe aortic stenosis 7 but seemingly rare in patients with lymphoproliferative derived aVWS. Current treatment guidelines per the International Society on Thrombosis and Haemostasis (ISTH) include Desmopressin (DDAVP), Factor eight/VWF concentrates, Intravenous Immune Globulin (IVIG), or plasmapheresis. When possible, correction of underlying disease process is particularly important [9]. In this report, we present a patient with aVWS due to relapsed CLL, complicated by gastrointestinal hemorrhage from angiodysplasia, treated with rituximab and venetoclax.

\section{Case Presentation}

Patient was a 64 year old male with history of CLL (genotype zap70 (+), CD38 (+), IgGVH, deletion 11, p53 (+)) in clinical remission following rituximab and fludarabine therapy (2007 to 2011) who presented to the emergency department with symptomatic anemia and melena. He had been followed in the Oncology clinic for rising leukocytosis since 2017 with cell counts consistently eighty to one hundred thousand. He had deferred repeat therapy due to an absence of symptoms. As demonstrated in Table 1, initial laboratory evaluation on admission was notable for hemoglobin of $6.7 \mathrm{~g} / \mathrm{dL}$, with elevated aPTT $(37.7 \mathrm{sec})$, normal PT $(13.2 \mathrm{sec})$, normal haptoglobin $(236 \mathrm{mg} / \mathrm{dL})$ negative coombs test and leukocytosis of $110 \times 10^{3} / \mathrm{mcL}$. PTT corrected on mixing study (44 second corrected to 33.8 with 1:1 mixing). Further labs obtained included negative lupus anticoagulant, factors VII (75\%), VIII (37\%), IX (97\%), XI (81\%) activity and von Willebrand factor panel (Von Willebrand factor Ag 21\%, Factor VIII Activity 37\%, Von Willebrand Factor Risocetin Cofactor Activity $<10 \%)$. His vWF panel was suggestive of Type 2 Von Willebrand disease in the absence of high molecular weight bands. Hospital course was significant for transfusion of 11 units of RBC and repeat endoscopic procedures (Figure 1). Initial upper endoscopy failed to identify a source of hemorrhage. Colonoscopy demonstrated a moderate volume of hematin without evidence of active hemorrhage. Transpyloric pill endoscopy revealed nonspecific hemorrhage throughout the small bowel. Repeat upper endoscopy was performed for continued transfusion requirements with identification of a jejunal angiodysplastic lesion managed with local injection of epinephrine and endoclipping. Despite this intervention, the patient continued to require daily $\mathrm{RBC}$ transfusion, necessitating attempted embolization
Ann Hematol Oncol - Volume 8 Issue 12 - 2021

ISSN : 2375-7965 | www.austinpublishing group.com

Heald et al. @ All rights are reserved
Citation: Heald JT and Oleszewski RT. Combination Venetoclax and Rituximab: A Novel Approach to Gastrointestinal Hemorrhage from Acquired Von Willebrand Syndrome in the Setting of Relapsed Chronic Lymphocytic Leukemia. Ann Hematol Oncol. 2021; 8(12): 1379. 


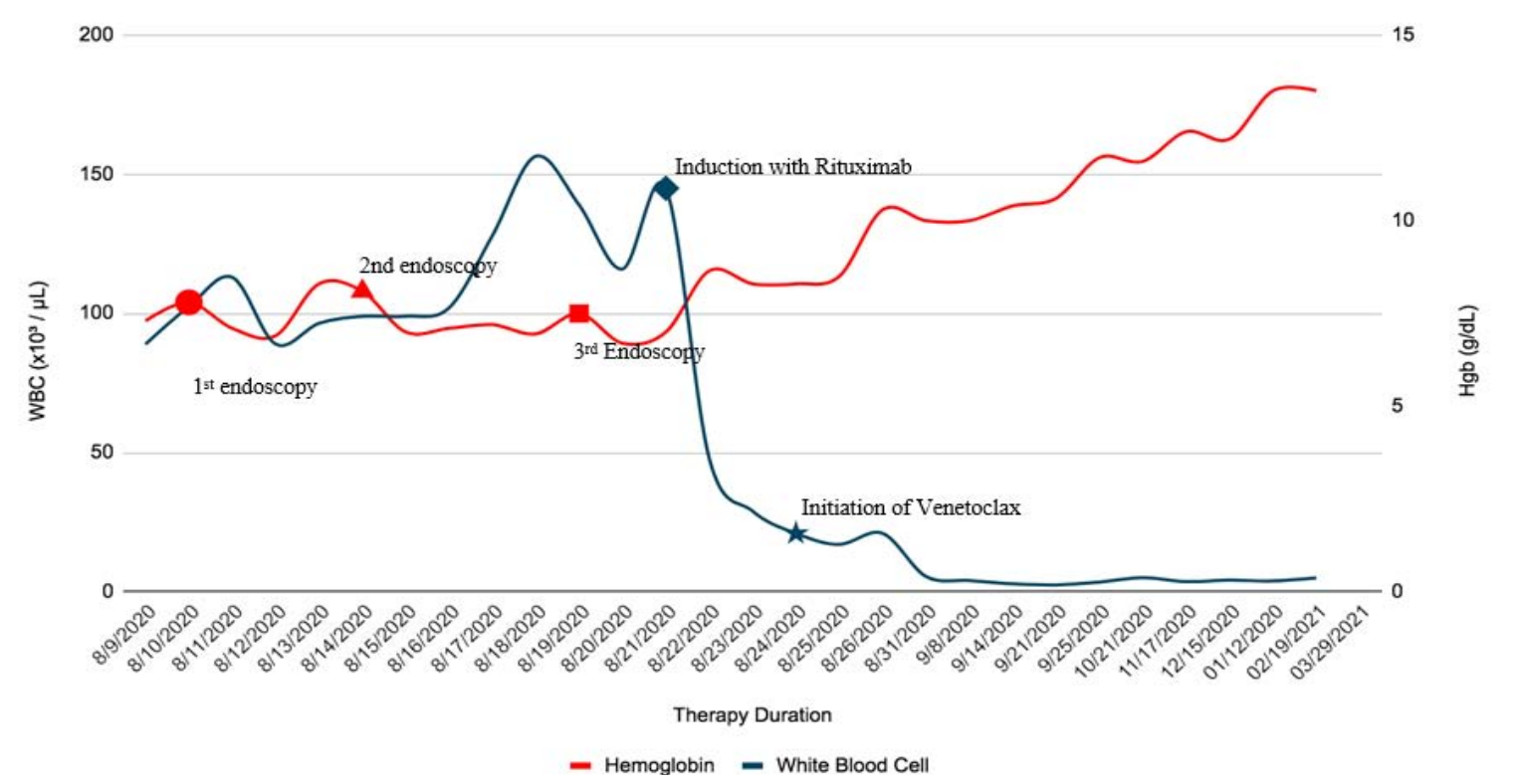

Figure 1: Demonstration of this patients trends in hemoglobin, white blood cell count in relation to treatment course. As shown above individual endoscopy procedures are denoted. Significantly, reduction of in white blood cell count corresponds with rise in hemoglobin and cessation of transfusion requirement.

Table 1: Initial laboratory values obtained on patient's admission relating to final diagnosis.

\begin{tabular}{|l|c|c|}
\hline \multicolumn{1}{|c|}{ Test/Result Name } & Result Values & $\begin{array}{c}\text { Reference } \\
\text { Range }\end{array}$ \\
\hline WBC $\left(\mathrm{X} 10^{3} / \mu \mathrm{L}\right)$ & 110 & $(4.0-10.3)$ \\
\hline Hemoglobin (g/dL) & 6.7 & $(12.0-16.2)$ \\
\hline Hematocrit (\%) & 22.3 & $(38.7-49.5)$ \\
\hline Platelet count (X10 $/ \mu \mathrm{L})$ & 198 & $(140-400)$ \\
\hline Protime (sec) & 13.2 & $(12.0-14.6)$ \\
\hline $\begin{array}{l}\text { Activated Partial Thromboplastin } \\
\text { time (sec) }\end{array}$ & 37.7 & $(23.7-35.0)$ \\
\hline INR & 1 & $(1.5-4.0)$ \\
\hline Factor VII (\%) & 75 & $(51-186)$ \\
\hline Factor VIII (\%) & 37 & $(60-140)$ \\
\hline Factor IX (\%) & 97 & $(60-150)$ \\
\hline Factor XI (\%) & 81 & $(50-200)$ \\
\hline VWF & High Molecular Weight & \\
\hline VWF Ristocetin Cofactor Activity & band (-) & $(50-200)$ \\
\hline Factor VIII Ag (\%) & $<10$ & \\
\hline VWF Antigen (\%) & 37 & $(140)$ \\
\hline
\end{tabular}

of the gastroduodenal artery. The procedure was aborted due to reversal of conventional directional flow. Given the high transfusion requirement he was initiated on Tranexamic acid and DDAVP for stabilization of the coagulopathy and hemorrhage. A final balloon assisted enteroscopy was significant for two oozing lesions in the gastric cardia, non-bleeding lesions in the lower gastric body, multiple non-bleeding lesions in the third/fourth portion of the duodenum, and the proximal jejunum all consistent with angiodysplasia (Figure 1). Once hemostasis was achieved, induction therapy with Rituximab followed by definitive treatment with rituximab and venetoclax for aVWS secondary to CLL began. Following induction, his leukocytosis markedly improved and gastrointestinal hemorrhage resolved (Figure 2). After discharge, he was followed in the oncology clinic and through six cycles of therapy, he had no recurrence of hemorrhage with resolution of anemia and stabilization of leukocytosis (Figure 2). Prior to cycle 4, Von Willebrand panel was repeated which showed normalization of activity (VWF ristocetin cofactor activity (60\%), VWF antigen (67\%) and normal factor VIII activity (73\%)).

\section{Discussion}

Acquired Von Willebrand Syndrome is a rare hemorrhagic disorder with laboratory findings that closely resemble those seen in congenital VWD though observed in patients without prior history of bleeding diathesis [9]. The laboratory evaluation is characterized by low levels of plasma factor VIII, Von Willebrand's factor and prolonged bleeding time [10]. In regards to hematologic malignancies, thirty to fifty percent of aVWS are secondary to Lymphoproliferative Disorders (LPD). The mechanisms thought to propagate this syndrome in LPD are either aberrant auto-antibodies directed against VWF or direct adhesion of high molecular weight VWF multimers onto malignant cells [3]. In situ vWF exists as a multifunctional glycoprotein that is crucial in proper coordination of primary and secondary hemostasis through mediation of platelet adhesion and intravascular transportation of coagulation factor VIII. A lesser-known function of VWF includes regulation of angiogenesis through activation of Vascular Endothelial Growth Factor Receptor-2 (VEGFR2) coupled with decreased sequestration of Angiopoietin-2 (Ang-2) [5]. The effects of these aberrancies has been demonstrated to increase angiogenesis and proliferation of neovascularization in endothelial cells derived from VWD patients and VWF-deficient mice (blood) [5].

As demonstrated in Table 1, this patient's laboratory evaluation was consistent with Type 2 Von Willebrand's Disease. Types 2A, 2B and $2 \mathrm{M}$ are associated with preferential loss or decreased function of high molecular weight multimers of the protein. These subtypes 


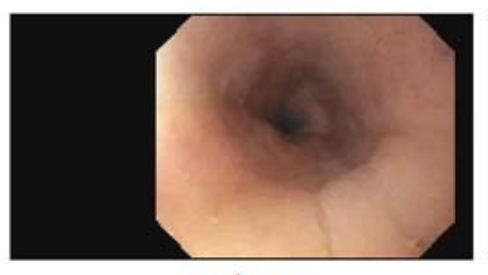

esophagus

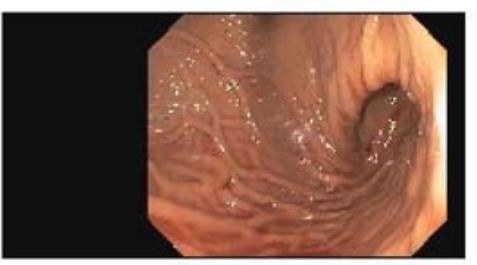

deformed stomach gastric angiodysplasia S/P APC

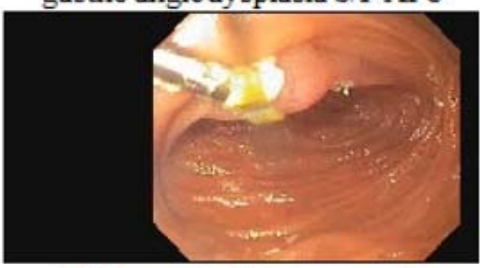

AVM noted adjacent near the previously placed endoclip

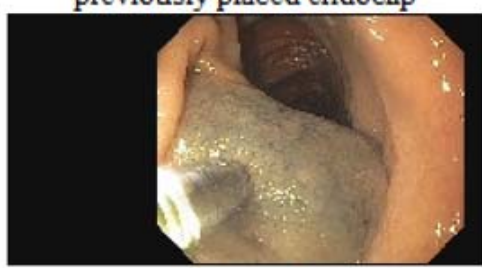

ink spot

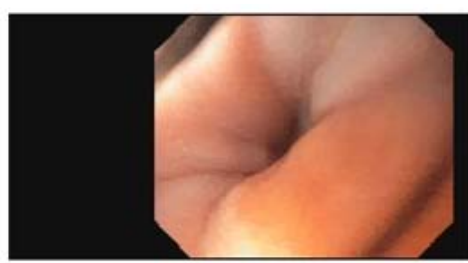

$1 \mathrm{~cm}$ salmon colored esophageal mucosa

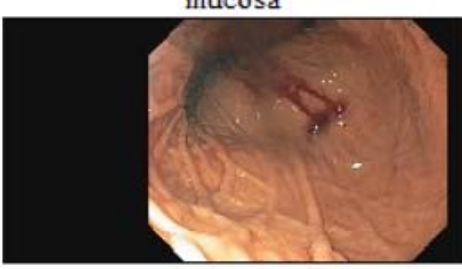

adjacent bleleding angiodsyplasia Entroscopy_0025.jpg

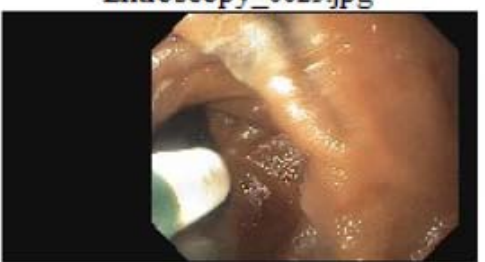

AVM treated with S/P APC

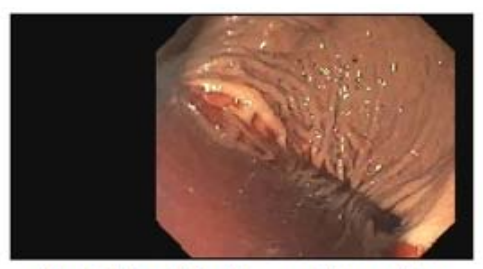

fresh blood in the gastric cavity

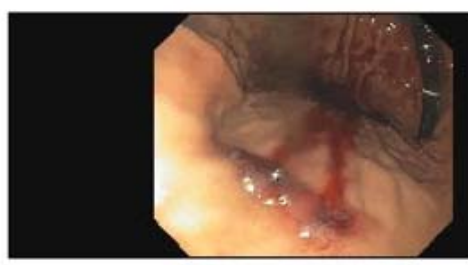

retroflex antrum with pylorus

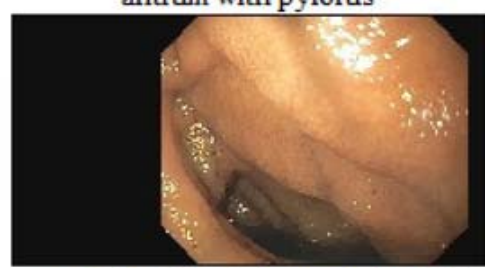

small AVM behind one of the fold pre and post intervention as well as non-active bleeding with the duodenum

are related to increased susceptibility to cleavage by ADAMTS13 (Type 2A), increased platelet adhesion and clearance via the reticuloendothelial system (type $2 \mathrm{~B}$ ) or gene variation resulting in dysfunction (Type 2M) [10]. This patient had no prior history of coagulopathy or hemorrhage and the bleeding diathesis corrected with treatment of CLL. Therefore, it is unlikely any of the specific subtypes were responsible for the clinical presentation. Due to the high clonal cell burden, the high molecular multimers were directly adhered to the cell surface and rendered non-functional. Antibody formation may also have contributed to this patient's disease, however Anti-vWF were not screened for during the hospitalization. Other etiologies deemed less probable included Heyde's syndrome, which presents as gastrointestinal hemorrhage due to loss of high molecular weight vWF multimers from shear stress in aortic stenosis or due to increased turbulence in LVADS7. This entity could not fully be excluded in this patient, as an echocardiogram was not obtained. Though absence of a murmur, heart failure or syncope significantly lowered the concern. Additionally hypothyroidism has been implicated in aVWS. In this patient a thyroid stimulating hormone was elevated $(11 \mathrm{mIU} / \mathrm{L})$ with normal free thyroxine $(0.8 \mathrm{ng} /$ dL) (T4) consistent with hypothyroidism. However, given temporal improvement and ultimate resolution of coagulopathy with treatment of CLL, hypothyroidism was determined to be an unlikely etiology.

This patient's initial chief complaint was for gastrointestinal hemorrhage in the setting of symptomatic anemia and endoscopy was deployed identifying multiple lesions consistent with angiodysplasia. The incidence of angiodysplasia itself is relatively rare, accounting for $4-7 \%$ of obscure gastrointestinal hemorrhage in the stomach and small intestine [12-14].

Upon establishing a high degree of certainty in the diagnosis of aVWS secondary to CLL, treatment was initiated per ISTH guideline recommendations9. Initially DDAVP and TXA were prescribed to obtain hemostasis; however, the gradation of anemia failed to improve significantly (Figure 1). Furthermore, DDAVP was discontinued due to development of significant hyponatremia. Despite society recommendations, IVIG was not attempted in this patient, instead opting for definitive treatment with rituximab and venetoclax for CLL. This resulted in a near immediate stabilization of hemoglobin with normalization of hemoglobin through six cycles of therapy (Figure 1).

Our case is not the first to report successful treatment of aVWS 
with rituximab however there does not appear to be a reported case of combination therapy with venetoclax. Venetoclax is a cytotoxic antineoplastic directed against cells with overexpression of BCL2. The combination of venetoclax and rituximab is currently FDA approved for treatment of relapsed or refractory CLL. Rituximab was first used successfully in the treatment of chronic gastrointestinal hemorrhage due to angiodysplasia from aVWS in a patient with an unidentified primary autoimmune disorder as reported by Hawkens et al. [8]. Additionally, Singh et al described success in a case series of four patients with IgG monoclonal gammopathy of undetermined significance [14]. Finally, rituximab has been shown to treat aVWS in one case of juvenile systemic lupus erythematosus [15]. Rituximab is postulated to works through cytotoxic reduction of $\mathrm{CD} 20$ positive $B$ cells producing anti-VWF antibodies [8]. In our patient, as these antibodies were not identified, restoration of normal vWF levels and activity presumably occurred from decreased consumption of vWF by clonal B cells. This is supported by the observation that hemorrhage and coagulopathy resolved with normalization of white blood cell count.

\section{Conclusion}

aVWS is a rare bleeding diathesis requiring a high degree of suspicion in order to consider as an etiology of refractory bleeding. Current guidelines recommend treating underlying pathology in order to achieve hemostasis however given its rarity, further treatments are not well established. This is the first recorded case of a patient with relapsed CLL complicated by aVWS with gastrointestinal hemorrhage from angiodysplasia successfully treated with this combination of therapy. Our case helps to further expand the available evidence for management of aVWS.

\section{References}

1. Rodeghiero F, Castaman G, Dini E. Epidemiological investigation of the prevalence of von Willebrand's disease. Blood. 1987; 69: 454-459.

2. Sadler JE, Mannucci PM, Berntorp E, Bochkov N, Boulyjenkov V, Ginsburg $\mathrm{D}$, et al. Impact, diagnosis and treatment of von Willebrand disease. Thromb Haemost. 2000; 84: 160-174.
3. Lavin M, Brophy TM, Rawley O, O'Sullivan JM, Hayden PJ, Browne PV, et al. Lenalidomide as a novel treatment for refractory acquired von Willebrand syndrome associated with monoclonal gammopathy. J Thromb Haemost. 2016; 14: 1200-1205.

4. Mital A. Acquired von Willebrand Syndrome. Adv Clin Exp Med. 2016; 25 1337-1344.

5. Starke RD, Ferraro F, Paschalaki KE, Dryden NH, McKinnon TA, Sutton RE et al. Endothelial von Willebrand factor regulates angiogenesis. Blood. 2011; 117: $1071-1080$

6. Randi AM, Laffan MA. Von Willebrand factor and angiogenesis: basic and applied issues. J Thromb Haemost. 2017; 15: 13-20.

7. Franchini M, Mannucci PM. Von Willebrand disease-associated angiodysplasia: a few answers, still many questions. Br J Haematol. 2013; 161: 177-182.

8. Hawken J, Knott A, Alsakkaf W, Clark A, Fayyaz F. Rituximab to the rescue: novel therapy for chronic gastrointestinal bleeding due to angiodysplasia and acquired von Willebrand syndrome. Frontline Gastroenterol. 2019; 10: 434437.

9. Federici $\mathrm{AB}$, Rand $\mathrm{JH}$, Bucciarelli $\mathrm{P}$, Budde $U$, Van Genderen $\mathrm{PJ}$, Mohri $\mathrm{H}$, et al. Scientific and Standardization Committee Communication Scientific Report of the Registry on Acquired von Willebrand Syndrome: Recommendations for Diagnosis and Management. 2000.

10. Jameson JL, Konkle BA. Disorder of Platelets and Vessel Wall. In Harrison's principles of Internal Medicine. 2018; 1: 828-830.

11. Gunnlaugsson O. Angiodysplasia of the stomach and duodenum. Gastrointest Endosc. 1985; 31: 251-254.

12. Clouse RE, Costigan DJ, Mills BA, Zuckerman GR. Angiodysplasia as a cause of upper gastrointestinal bleeding. Arch Intern Med. 1985; 145: 458461.

13. Moretó M, Figa M, Ojembarrena E, Zaballa M. Vascular malformations of the stomach and duodenum: an endoscopic classification. Endoscopy. 1986; 18 : 227-229.

14. Singh P, Siegel J, Caro J, et al. Rituximab use in four patients with acquired von Willebrand's syndrome. Blood. 2006; 108: 4045.

15. Jimenez AR, Vallejo ES, Cruz MZ, Cruz AC, Miramontes JV, Jara BS. Rituximab effectiveness in a patient with juvenile systemic lupus erythematosus complicated with acquired Von Willebrand syndrome. Lupus. 2013; 22: 1514-1517. 